\title{
Understanding atom movement during lateral manipulation with the STM tip using a simple simulation method
}

\author{
A. Kühnle * , G. Meyer, S.W. Hla, K.-H. Rieder \\ Fachbereich Physik, Freie Universität Berlin, Arnimallee 14, 14195 Berlin, Germany
}

Received 3 September 2001; accepted for publication 29 November 2001

\begin{abstract}
We report on a fast simulation method to investigate the movement of an atom induced by the tip during lateral manipulation with a scanning tunneling microscope. The simulation is based on a model assuming the atom moving in the combined potential of tip and surface. The pathway of the tip is subdivided in small steps, and the atomic position for each step is calculated by an iterative algorithm searching for the closest energetic minimum. The method is demonstrated for manipulation on the (1 111$)$ surface of an fcc metal. Our model calculations predict which energetic minima of the surface are attained by the atom during manipulation. The details of the modelled manipulation curves allow a precise description of the atomic pathway in dependence on manipulation direction and positioning of the tip relative to the atom. Furthermore, the simulation predicts a transition from the so-called pulling to sliding manipulation mode upon reducing tip-surface distance, well in agreement with general experimental observations. To test our

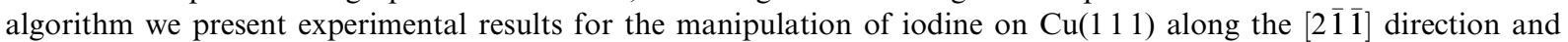
compare them to simulated manipulation curves. The comparison allows for a complete understanding of all details in atomic movements during manipulation along a complicated path. (c) 2001 Published by Elsevier Science B.V.
\end{abstract}

Keywords: Scanning tunneling microscopy; Single crystal surfaces

\section{Introduction}

Lateral manipulation of single atoms and molecules allows the build-up of nano-structures in an atom by atom way. The possibility to assemble small structures with atomic precision offers a va-

\footnotetext{
${ }^{*}$ Corresponding author. Present address: Institute of Physics and Astronomy, University of Aarhus, DK-8000 Aarhus, Denmark. Tel.: +45-8942-3691; fax: +45-8612-0740.

E-mail address: kuehnle@ifa.au.dk (A. Kühnle).
}

riety of new experiments on the atomic scale. Until now, structures with up to 100 atoms have been formed [1,2], and it has been demonstrated that a variety of adsorbates and even substrate atoms can be moved to desired positions by lateral manipulation $[3,4]$. Also larger molecules such as diiodobenzene, porphyrin or $\mathrm{C}_{60}$ have been manipulated [5-7]. By recording the tip height (feedback loop signal) or the tunneling current during the manipulation process, detailed information on the adsorbate movement can be gained in real time [8]. The atom-by-atom assembly of larger structures is, 
however, very time consuming, demonstrating a serious drawback of the scanning tunneling microscope (STM) manipulation for future applications. Obviously, an automation of at least parts of the manipulation process is desirable for advanced applications. One step in this direction is a better understanding and interpretation of the feedback loop signal during manipulation. For a detailed quantitative understanding of the data quite elaborate theoretical work has to be performed $[9,10]$. However, these calculations are very time consuming and, therefore, limited in real time implementation.

Here we present a simple simulation method, operating rather fast and yielding a good qualitative understanding of the tip height curves or tunneling current signals. The optimum path for atom movement, using the smallest necessary force, can be easily predicted by these simulations. This is an important issue, since any excess strain on the tip apex could result in its destruction.

In an STM manipulation experiment performed in constant current mode the change in tip height gives insight into the processes during the lateral manipulation [8]. Monitoring the change in tip height exhibits a significant advantage compared to other techniques for controlling the manipulation process, since the tip height curves immediately show the success of the manipulation. Thus, the simulation should provide information corresponding to these tip height curves obtained by the experiment. Since the calculations are performed in constant height mode, the change in tunneling current is monitored as in many theoretical investigations on STM, therefore, the manipulation curves presented here show changes in tunneling current instead of the tip height. This approach is possible because the tunneling current signal in constant height mode offers a similar information as the tip height in constant current mode, while simulating with constant tip height reduces the computation time remarkably.

\section{Simulation method}

The main idea of the presented simulation is to model the overall potential energy surface formed by both, STM tip and substrate surface. In this potential, the adsorbate, considered as a spherically shaped, metal-like atom, moves without the action of any other force. The tip is moved in constant height over the surface along a given path in small steps. After each step, the most probable adsorbate position is determined by searching for the closest local potential minimum thereby assuming the hollow site as the favorable adsorption position. The influence of temperature is included using a Boltzmann distributed probability for the adsorbate to overcome potential barriers.

\subsection{Surface and tip potential}

The surface is assumed to be a continuous, sinusoidal potential with the hexagonal symmetry of the (1 111 ) surface of an fcc crystal. Such a potential can be achieved by a superposition of three sinusoidal waves:

$$
\begin{aligned}
U_{\text {surf }}(x, y)= & \frac{U_{0}}{3}\left\{\cos \left(\left(x-\frac{y}{\sqrt{3}}\right) \frac{2 \pi}{d}\right)\right. \\
& +\cos \left(\left(x+\frac{y}{\sqrt{3}}\right) \frac{2 \pi}{d}\right) \\
& \left.+\cos \left(2 x \frac{2 \pi}{d}\right)\right\}
\end{aligned}
$$

In this equation, $U_{0}$ is the potential amplitude and $d$ the lattice constant of the fcc crystal. Two kinds of hollow sites can be identified on the fcc (1 111 ) surface, namely fcc and hcp, depending of the absence or existence of a substrate atom in the second layer underneath the hollow site. Since the second layer potential is not included, the simulation is not differentiating between fcc and hcp hollow sites.

The tip potential has to fulfil the following two conditions. First, it has to provide a wide range attractive potential to model the attraction between two metal atoms until they reach point contact. Second, in order to simulate the point contact, a repulsive force has to be included for very short tip-adsorbate distances. A Morsepotential is known to meet both constraints.

$U_{\text {tip }}\left(r, r_{0}\right)=D\left(1-\exp \left\{-\sqrt{\frac{K}{D}}\left(r-r_{0}\right)\right\}\right)^{2}$ 
For this potential, the equilibrium position is given by $r_{0}$ and the position of the adsorbate by $r$. The constant $K$ corresponds to the spring constant of a harmonic oscillator for the truncated expansion of the Morse-potential, and $D$ equals to the potential at infinite tip-adsorbate distance, whereas the potential energy for the equilibrium position, $U\left(r_{0}\right)$, is set to zero. For distances $r<r_{0}$, the potential becomes repulsive, otherwise it is attractive. The characterisation of the substrate and tip potentials with these simple formulas involves, of course, several simplifications and approximations. Special tip conditions or adsorbate shapes are neglected. Furthermore, the surface potential as well as the tip potential are assumed to be independent on the tip height, although, in fact, the surface potential is known to be influenced slightly by the presence of the tip and vice versa which is of importance for some systems. The complete description of these effects is beyond the simple model used in this paper. The present approach reveals, however, a straight-forward, qualitative understanding of the experimental situation, while keeping the computational effort at minimum.

\subsection{Temperature}

Temperature effects are taken into account by including a Boltzmann-probability to overcome potential barriers. This is realised using a Metropolis-algorithm [11], which weights the new atom position with energy $E_{i+1}$ with a probability $p_{i, i+1}$ depending on the energy of the previous atom position, $E_{i}$ :

$p_{i, i+1}=1, \quad E_{i+1}-E_{i}<0$

$p_{i, i+1}=\exp \left\{-\left(E_{i}-E_{i+1}\right) / k T\right\}, \quad E_{i+1}-E_{i}>0$

For each step of the tip, the computation of an adequate number of adsorbate steps is necessary to guarantee the equilibrium of the process. This corresponds to a higher adsorbate velocity compared to the velocity of the tip in accordance with the experimental conditions. By changing the number of adsorbate steps after one tip step, the influence of different manipulation velocities is easily taken into account.
With the calculated adsorbate position, the distance between tip apex and adsorbate, $z$, can be determined, giving a value for the tunneling current, since the tunneling current is exponentially dependent on the tip-sample distance.

$I \propto \exp \{-A \sqrt{\Phi} z\}$

With this equation, where $\Phi$ is the metal work function and $A=2 \sqrt{2 m_{e}} / \hbar$, the tunneling current is calculated in arbitrary units.

\section{Results}

\subsection{Transition from pulling to sliding}

In the following, we first briefly describe the general features of manipulation curves before the simulation results are presented. For an attractive interaction between tip and adsorbate, the experimental manipulation is performed either in pulling or sliding mode. The manipulation curve is continuous for the latter, while it is discontinuous for the former mode, as described in [8]. In the case of small molecules and attractive forces, the third manipulation mode, pushing, is only rarely observed, mainly for manipulations along step edges. While increasing the strength of interaction by reducing the tip height, the manipulation mode is changing from pulling to sliding. This is due to the fact that the adsorbate is mainly following the substrate minima in the case of weak tip-adsorbate interaction resulting in the pulling mode, exhibiting the discontinuities of the adsorbate jumps. In the case of small tip heights and therefore strong tip-adsorbate interactions, the influence of the substrate potential becomes more and more negligible and the adsorbate is straightly following the tip. The result is the continuous sliding mode. These experimental findings [12] are very well reproduced by the simulation. The hexagonal substrate surface and one path of the tip is shown in Fig. 1a. The simulated tunneling current curves for this situation are shown in Fig. 1b, where the tip height is gradually decreased by about $6 \%$ from case 1 , which is a pulling curve, to case 4 showing the sliding mode. The amplitude in the tunneling 

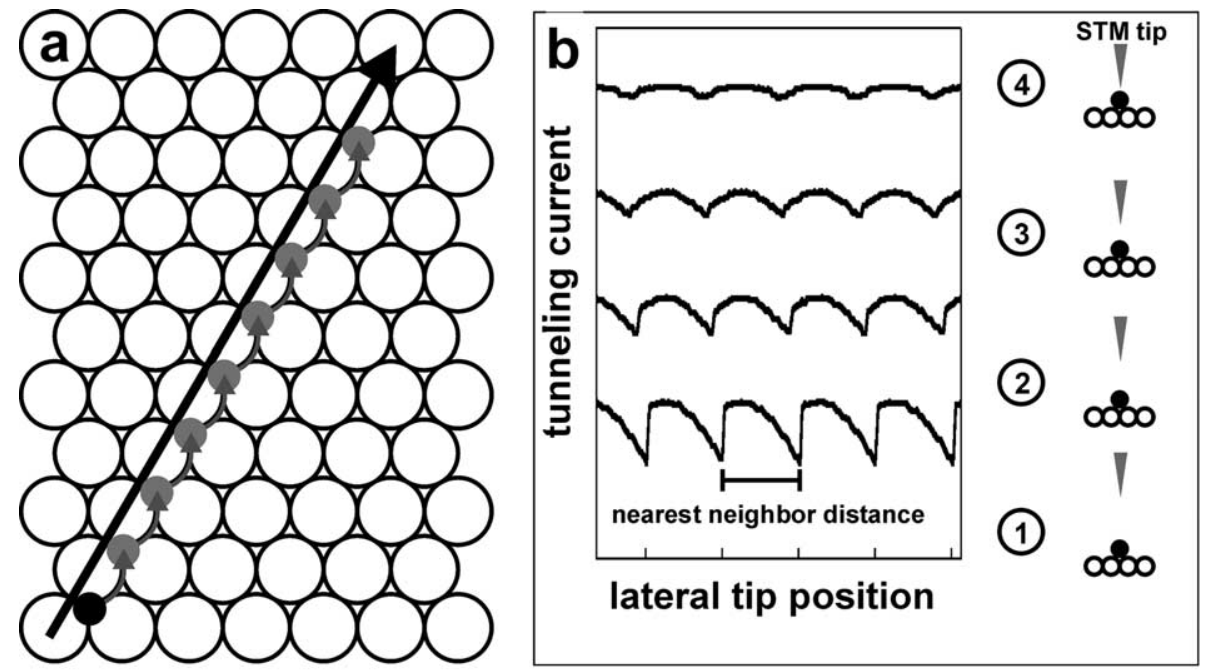

Fig. 1. Transition from pulling to sliding while decreasing the tip height. (a) Sketch of the manipulation geometry. The tip is drawn directly on top of a close-packed row (black arrow). The adsorbate is following the tip, visiting the substrate minima beside the tip path as indicated by the circles. (b) Tunneling current curves are shown over the lateral tip position. From 1 to 4 the tip height is decreased by $6 \%$. With decreasing tunneling distance the interaction between tip and adsorbate increases which leads to (i) a change of the manipulation mode from pulling to sliding and (ii) to an increase in total tunneling current. The overall change in tunneling current, $\Delta h$, is higher for the pulling mode than for the sliding mode which indicates a weaker interaction for the pulling than the sliding mode.

current, $\Delta h$, is related to the strength of the tipadsorbate interaction [10]. For a strong tipadsorbate interaction, the amplitude is small as seen in the sliding curves. For the pulling mode, the interaction is weak and $\Delta h$ becomes larger, which is evidently reproduced in Fig. 1b.

\subsection{Dependence on different starting points of the manipulation path}

During the manipulation, the change in the tunneling current indicates the distances between the adsorption sites adopted by the adsorbate. For the ease of discussion we assume arbitrarily the starting position of the atom to be an fcc site. If the tip is drawn directly over a closed-packed row, the adsorbate jumps only in the fcc hollow sites. Thus, the distances shown in the manipulation curve correspond to the nearest neighbor distance of the crystal as marked in Fig. 1.

Varying the manipulation by shifting the starting point of the tip way relative to the adsorbate results in different adsorbate movements which can be deduced directly from the manipulation curves.
For the simulations described next, the manipulation angle is kept constant while the tip starting position path is gradually shifted relative to the adsorbed atom as shown in Fig. 2. On the left side, the tip path and the movement of the atom is shown. In the topmost path (a) the tip is moved directly over a close-packed row, whereas in the lowest path (c) the starting position of the tip has been shifted and is exactly in between two substrate atoms. An intermediate manipulation path is displayed in (b). On the right hand side, the corresponding manipulation curves are presented. The topmost manipulation curve (a) exhibits the characteristic shape of the pulling mode, it shows discontinuities separated by the nearest neighbor distance $d$. Upon gradually shifting the tip starting position towards the position between two substrate atoms, the manipulation curves show significant changes from pulling steps with the nearest neighbor distance $d$ to steps of the distance $d / 2$, while at the same time the amplitude, $\Delta h$, of the tunneling current is decreasing. The first observation is due to the fact that the adsorbate is adopting only fcc hollow sites for the first case, 

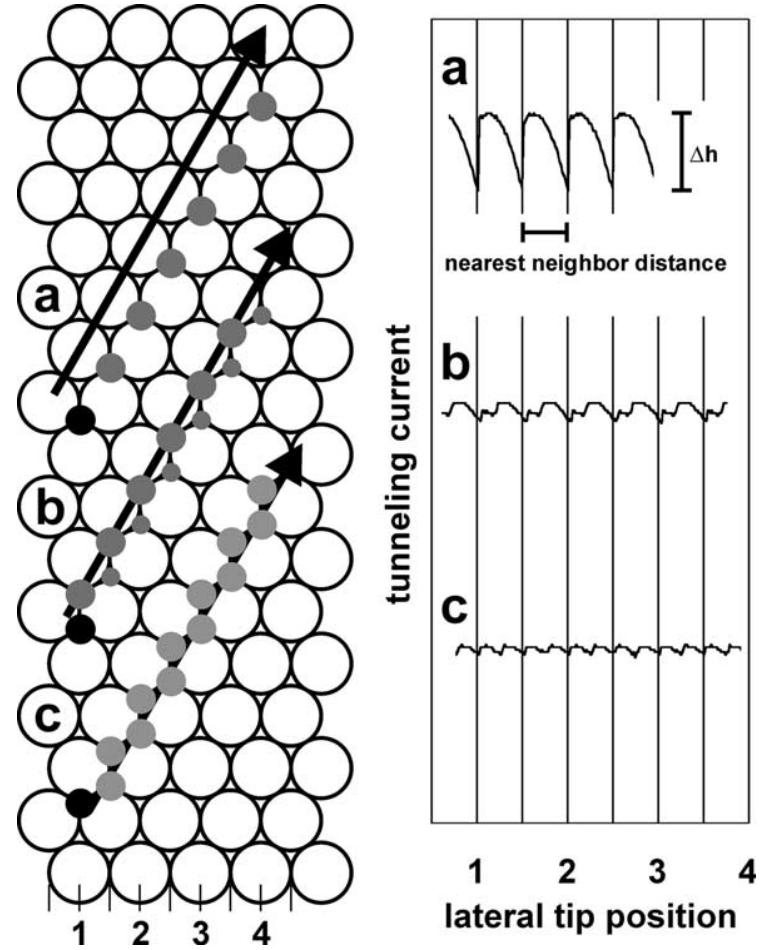

Fig. 2. Manipulation geometry and corresponding tunneling current curves for three different tip paths. The curves are displayed with an offset. (a) shows the same curve as in Fig. 1. For (b) and (c), the starting point of the tip has been shifted relative to the adsorbed atom. This results in a change in the manipulation curve due to the fact that additional substrate minima are transiently visited by the atom in (b). The shorter remain time is sketched by circles with smaller radii. In the last case (c) the two different substrate minima are equally visited leading to equal peaks in the tunneling current curves with half the distance between the peaks as compared to (a).

while it shortly remains also in the hep minima when the manipulation path is shifted. (The shorter remaining time is indicated by the circles with smaller radii in Fig. 2.) Therefore, additional peaks arise in the tunneling current curve (b). For the last case (c), the two substrate minima are visited equally and the difference in the tunneling current peaks is vanished. The decrease in amplitude, $\Delta h$, is caused by an increase in the interaction between tip and adsorbate from situation (a) to (c). From this it is possible to conclude that the mean tip sample distance is smaller in the latter case, while it is larger when the adsorbate stays only in one kind of adsorption sites.

\subsection{Dependence on the manipulation angle}

Similar to the dependence on the parallel shift of the manipulation path, the manipulation curve also depends on the manipulation angle. By rotating the manipulation direction, the tip is passing over both types of minima, fcc and hcp, and the adsorbate is trying to follow the tip as close as possible while at the same time staying in the substrate minima [13]. Therefore, the adsorbate adopts both minima which results in a change to smaller distances between the peaks in the manipulation curve. This is shown in Fig. 3 where results for two different manipulation angles are presented. In Fig. 3a the manipulation starts with longer jumps until the other type of minima is also visited, resulting in additional discontinuities in the tunneling current curve. In Fig. $3 b$ the adsorbate mainly performs short distance jumps, apart from two long distance jumps needed to remain at the tip. These long distance jumps are clearly observed in the tunneling current curves. When performing long distance jumps, the interaction becomes weaker, resulting in a large amplitude, while the amplitude remains small in the case of strong tip-adsorbate interaction.

\subsection{Analysis of experimental data: manipulation along the $[2 \overline{1} \overline{1}]$ direction}

The simulation method is finally applied to determine the precise path of an adsorbate during an experimental manipulation. The lateral manipulation has been performed with a single iodine atom, which is pulled along the $[2 \overline{1} \overline{1}]$ direction on a $\mathrm{Cu}\left(\begin{array}{lll}1 & 1 & 1\end{array}\right)$ surface. The STM images before and after the manipulation and the corresponding lateral manipulation curve are shown in Fig. 4. In Fig. 4a the iodine atom is on the left side of the image, and an arrow indicates the manipulation path. Fig. $4 \mathrm{~b}$ is recorded after the manipulation. The iodine atom has been moved to the right. The structure of the manipulation curve displayed in Fig. $4 \mathrm{c}$ is more complicated than the previously measured curves which has been performed along a close-packed row direction [8]. In the following, we first discuss the experimental details. Then we extract the characteristic distances from the curve 

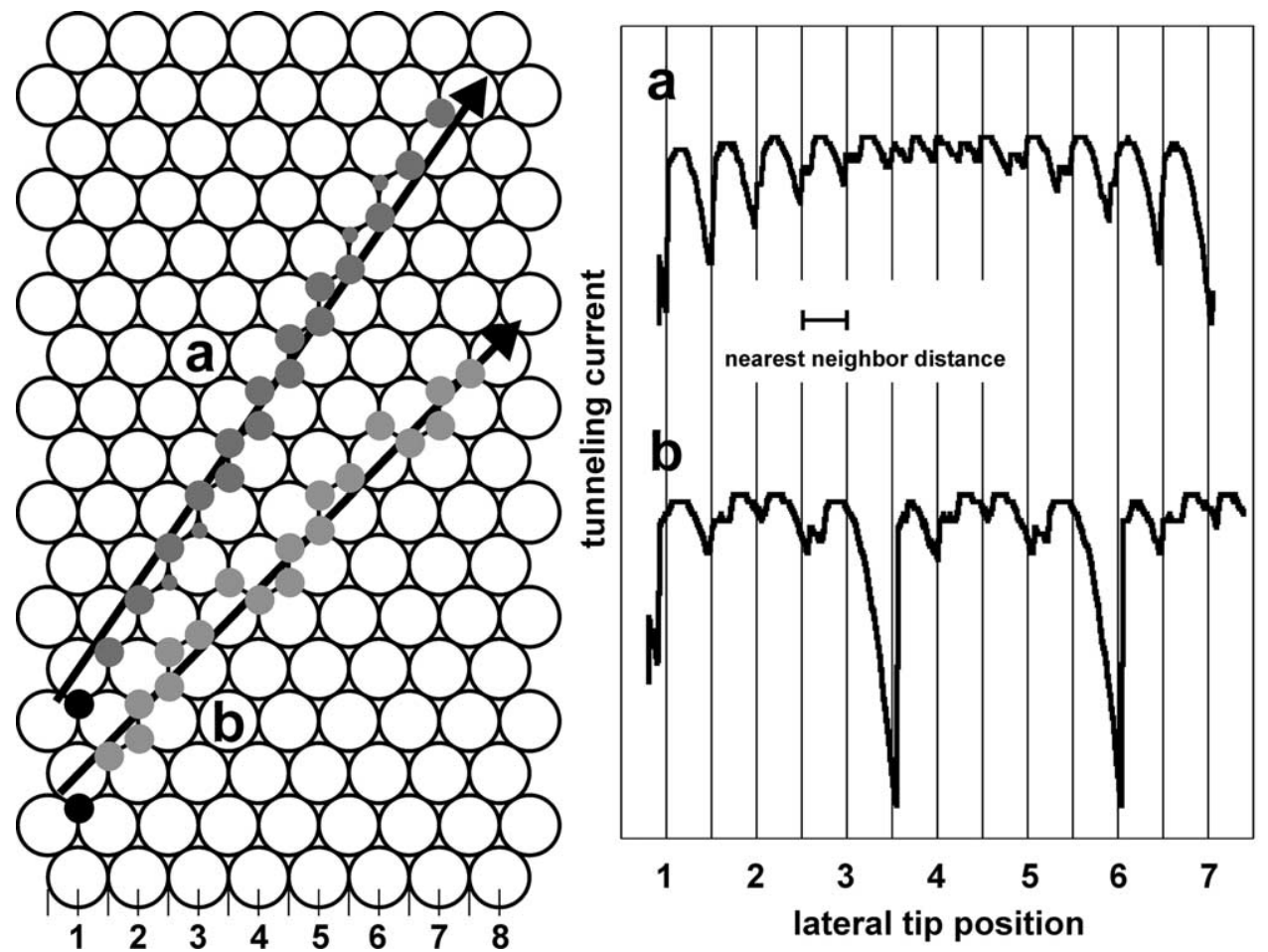

Fig. 3. Manipulation path and tunneling current curve for two different manipulation paths rotated with respect to the close-packed row. The left figure shows the path of the tip and the resulting movement of the adsorbate. Transiently visited substrate minima are displayed by small circle radii. In the right graphs the corresponding tunneling current curves are shown. The tunneling current curve (b) clearly exhibits two long distance jumps performed by the atom.

and finally use the simulation to understand the characteristic shape of the curve.

The manipulation starts by defining the desired start- and end-point. First, the tip is scanned forth and back between both points with the same tunneling parameters as used for imaging. This produces two of the upper curves in Fig. 4c lying nearly on top of each other with a peak on the left side, indicating the initial position of the iodine atom. Then the tip is again at the starting position and the manipulation is initiated by reducing the tunneling voltage, resulting in a decrease of the tip height of about $2 \AA$. The tip is moved over the adsorbate while the change in tip height is monitored. This is shown in the lower line in Fig. 4c, the actual manipulation curve. At the end-point of this manipulation the tip is drawn back to its original height. The final position of the iodine atom can be seen in the third upper curve indicated by the peak on the right side.
The experimental manipulation curve exhibits a periodicity with a characteristic distance of $4.42 \AA$, which is marked by thin vertical lines. A sketch of the periodic structure is shown in the insert of Fig. 4c. It consists of two maxima, which are separated by distances of 1.47 and $2.94 \AA$, respectively. The ratio between these distances is $1: 2$, which equals the ratio between the distances between fcc and hep sites along the $[2 \overline{1} \overline{1}]$ direction. In addition, a phase shift is observable in the manipulation curve. Grey bars indicate parts in the manipulation curve where the periodic pattern shifts. This is shown by the black arrows, which each are pointing at the beginning of a periodic unit as displayed in the insert. The position of the arrow in the middle is shifted by $2.21 \AA$ with respect to the thin lines.

To understand the mentioned details during the manipulation, a simulation along the $[2 \overline{1} \overline{1}]$ direction was performed (Fig. 5). For the manipulation 


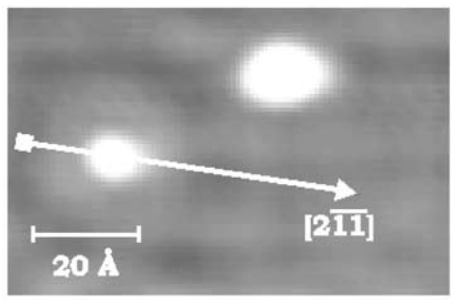

a

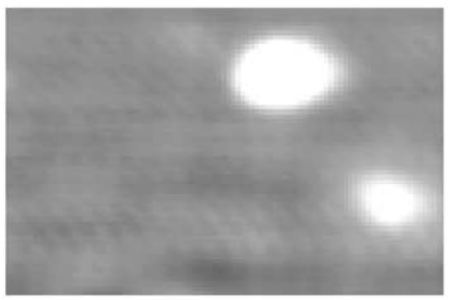

b

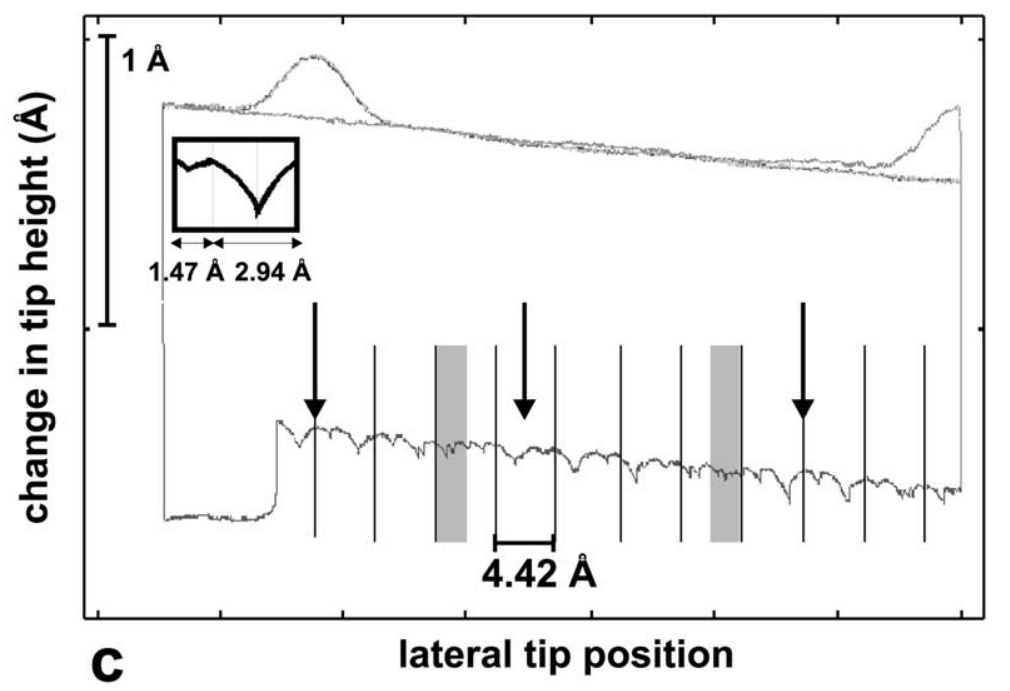

Fig. 4. Experimental iodine manipulation curve on $\mathrm{Cu}(111)$. (a) STM image before manipulation of iodine along the $[2 \overline{1} \overline{1}]$ direction. The white arrow indicates the manipulation direction. (b) STM image after manipulation. The iodine has moved from the upper left to the lower right part. (c) Tip height curve for the iodine manipulation. The upper lines are due to pre-scans before and after the manipulation with normal imaging parameters. The lower curve is the measured change in tip height during manipulation. The periodic units with a distance of $4.42 \mathrm{~A}$ are separated by grey lines, and a sketch of the periodic unit is shown in the insert. Two occurring phase shifts are indicated by grey areas. Black arrows are pointing at the beginning of three periodic units, and it can been seen that the positions of the arrows with respect to the thin grey lines are shifted.

directly along the $[2 \overline{1} \overline{1}]$ direction, the simulated tunneling current signal exhibits clear discontinuities. These are due to long jumps of the adsorbate around the substrate atoms without remaining in the passed substrate minima, as indicated with the long curved arrows in Fig. 5a. Since the experimental curve displayed in Fig. 4c rarely shows discontinuities, the path of the iodine is usually different from this situation.

For a small rotation of the manipulation direction the adsorbate starts to perform smaller jumps, remaining in additional substrate minima (Fig. $5 b)$. This is reflected by the change in the tunneling current curve, which becomes continuous. One exception is the first jump in Fig. 5b, where the adsorbate is still avoiding the passed minima.

From these simulation results it is possible to determine the complete iodine movement in detail. Fig. 5c shows a sketch of the experimental situation. The adsorbate is moving around the substrate atoms, visiting all passed substrate minima, instead of jumping over them. Because of this, the tip height curve shows mainly a continuous movement with the characteristic shape displayed in the insert in Fig. 5c. From time to time the atom has to jump into the neighboring row to follow the 

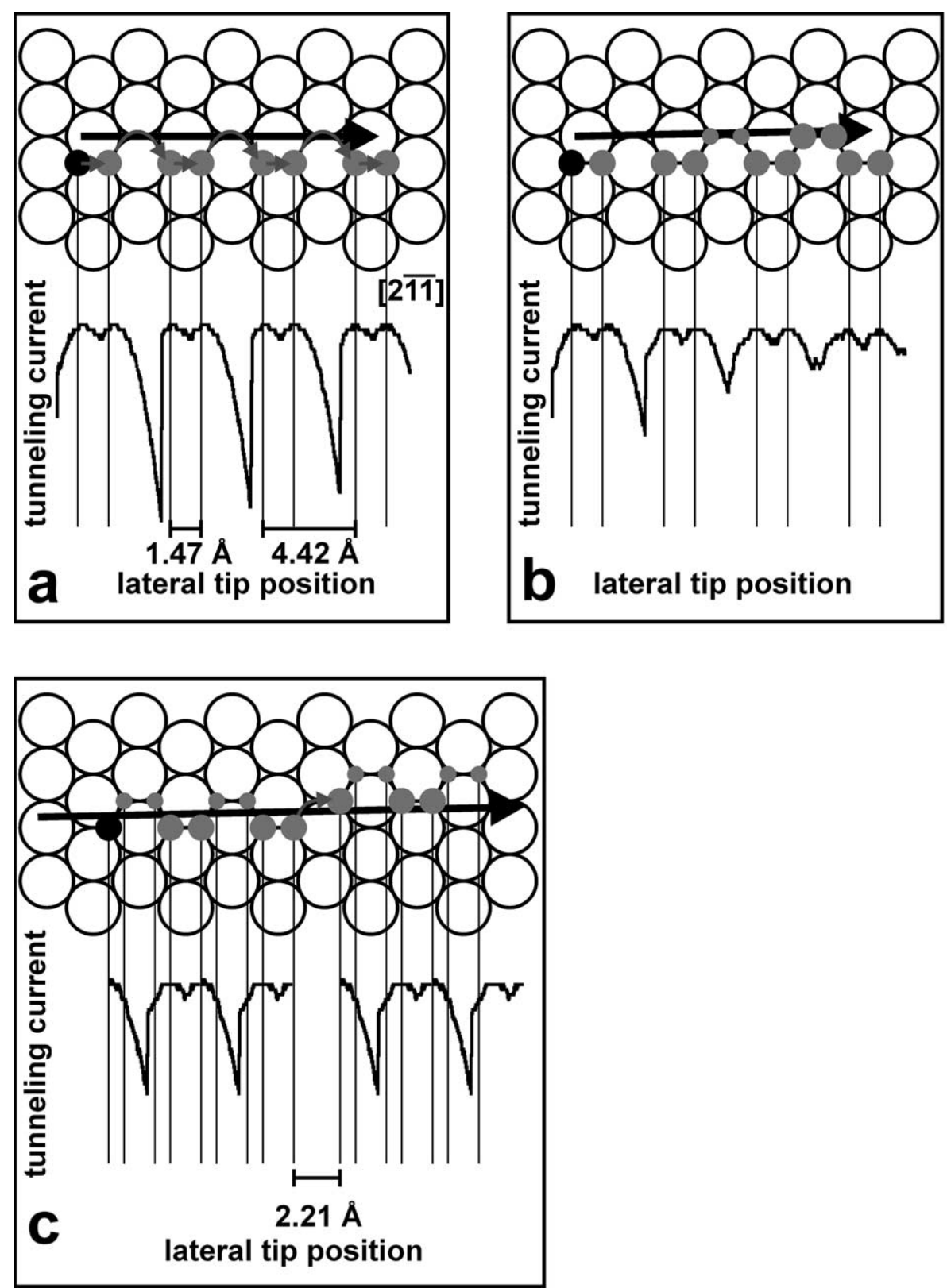

Fig. 5. Simulation of the manipulation along the $[2 \overline{1} \overline{1}]$ direction. (a) Manipulation along the $[2 \overline{1} \overline{1}]$ direction. In this case the simulation results in proper jumps over the substrate atoms, which corresponds to a discontinuous current signal. (b) The manipulation direction is rotated by $1.2^{\circ}$. Now the adsorbate is transiently visiting additional substrate minima. This leads to a change in the tunneling current signal, which becomes continuous. (c) The complete simulation of the experimental data presented in Fig. 4 . A jump of the iodine into a neighboring row yields a shift of $2.21 \AA$, explaining the observed phase shift in the experimental curve in Fig. $4 \mathrm{c}$.

tip path causing the observed phase shift of $2.21 \AA$ in the tip height signal. From this it is possible to conclude that the experimental curve presented in
Fig. $4 \mathrm{c}$ has been performed with a small angle with respect to the $[2 \overline{1} \overline{1}]$ direction, and we can precisely determine the substrate minima visited by the 
iodine atom. The observed phase shifts are due to a jump of the adsorbate into the neighboring row.

\section{Conclusions}

We have presented a simple and fast simulation program to generate qualitative manipulation curves in dependence on tip height and manipulation directions. The basic manipulation modes pulling and sliding and their dependence on the tip-adsorbate interaction strength were simulated. The transition from pulling to sliding while gradually approaching the surface with the tip is in good agreement with experimental results. Furthermore, additional features due to varying manipulation angles and starting positions of the manipulation path can be simulated in detail. Apart from simulating the general features of manipulation curves, we have applied our simulation program to a more complex case of iodine manipulation along the $[2 \overline{1} \overline{1}]$ direction on a $\mathrm{Cu}-$ (111) surface for direct comparison with experimental data. The characteristic distances as well as the specific shape of the manipulation curve could be well reproduced and understood, gaining further insight into the detailed manipulation process.

\section{References}

[1] D.M. Eigler, E.K. Schweizer, Nature 344 (1990) 524.

[2] M.F. Crommie, C.P. Lutz, D.M. Eigler, E.J. Heller, Physica D 83 (1995) 98.

[3] G. Meyer, S. Zöphel, K.-H. Rieder, Phys. Rev. Lett. 77 (1996) 2113.

[4] J.T. Li, W.-D. Scheider, R. Berndt, Appl. Phys. A 66 (1998) S675.

[5] S.W. Hla, A. Kühnle, L. Bartels, G. Meyer, K.-R. Rieder, Surf. Sci. 454-456 (2000) 1079.

[6] J.K. Gimzewski, T.A. Jung, M.T. Cuberes, R.R. Schlittler, Surf. Sci. 386 (1997) 101.

[7] H. Tang, M.T. Cuberes, Ch. Joachim, J.K. Gimzewski, Surf. Sci. 386 (1997) 115.

[8] L. Bartels, G. Meyer, K.-H. Rieder, Phys. Rev. Lett. 79 (1997) 697.

[9] X. Bouju, Ch. Girard, Phys. Rev. B 55 (1997) 16498.

[10] X. Bouju, Ch. Joachim, Ch. Girard, Phys. Rev. B 59 (1999) R7845.

[11] W.H. Press, B.P. Flannery, S.A. Teukolsky, W.T. Vetterling, Numerical Recipes, Cambridge University Press, Cambridge, 1998.

[12] A. Kühnle, G. Meyer, S.W. Hla, K.-H. Rieder, unpublished results.

[13] L. Bartels, G. Meyer, K.-H. Rieder, Chem. Phys. Lett. 285 (1998) 284. 